\title{
The Effect of Predicted Vehicle Displacement on Ground Crew Task Performance and Hardware Design
}

\author{
Laura Ashley Atencio* and David W. Reynolds. ${ }^{\dagger}$ \\ NASA Marshall Space Center, AL, 35812
}

\begin{abstract}
NASA continues to explore new launch vehicle concepts that will carry astronauts to lowEarth orbit to replace the soon-to-be retired Space Transportation System (STS) shuttle. A tall vertically stacked launch vehicle ( $\geq 300 \mathrm{ft}$ ) is exposed to the natural environment while positioned on the launch pad. Varying directional winds and vortex shedding cause the vehicle to sway in an oscillating motion. Ground crews working high on the tower and inside the vehicle during launch preparations will be subjected to this motion while conducting critical closeout tasks such as mating fluid and electrical connectors and carrying heavy objects. NASA has not experienced performing these tasks in such environments since the Saturn V, which was serviced from a movable (but rigid) service structure; commercial launchers are likewise attended by a service structure that moves away from the vehicle for launch. There is concern that vehicle displacement may hinder ground crew operations, impact the ground system designs, and ultimately affect launch availability. The vehicle sway assessment objective is to replicate predicted frequencies and displacements of these tall vehicles, examine typical ground crew tasks, and provide insight into potential vehicle design considerations and ground crew performance guidelines. This paper outlines the methodology, configurations, and motion testing performed while conducting the vehicle displacement assessment that will be used as a Technical Memorandum for future vertically stacked vehicle designs.
\end{abstract}

\section{Introduction}

A tall launch vehicle ( $\geq 300 \mathrm{ft}$ ) is exposed to the natural environment while positioned on the pad at the launch site. (Figure 1 presents a height comparison between the a) Saturn V, b) shuttle, and c) Ares I vehicles.) Varying directional winds and vortex shedding will cause the vehicle to sway in an oscillating motion. ${ }^{1}$

The Human Factors (HF) Engineering (HFE) discipline is responsible for HFE design requirements for NASA MSFC projects/programs and for promoting sound design principles to support optimal and safe performance of ground operations. Application and adherence to HFE processes and requirements promotes supportability, operability, and sustainability of these projects/programs and lowers the life cycle costs of operations while supporting safety and mission success.

\footnotetext{
* Co-Op, Marshall Space Flight Center, The University of Alabama in Huntsville, Huntsville, AL, 35899.

$\dagger$ Human Factors Engineer, Stage Analysis Branch, Marshall Space Flight Center, AL 35812.
} 


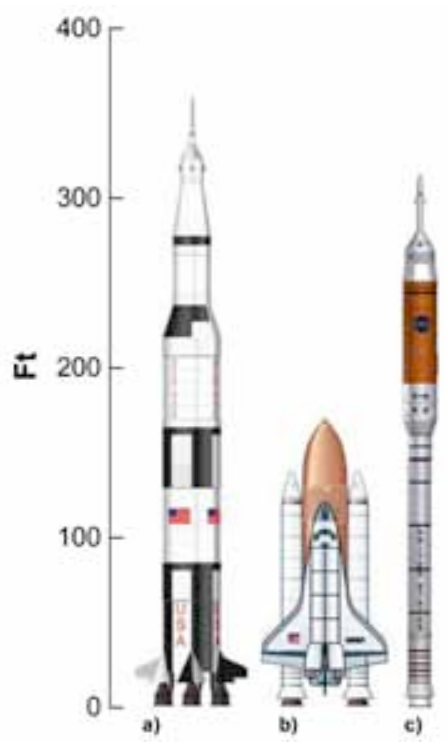

Figure 1. Height comparison between the Saturn V, shuttle, and Ares I vehicles.

NASA Marshall Space Flight Center's (MSFC's) HF team recognizes that the vehicle sway may hinder ground crew operation, impact the ground system designs, and ultimately affect launch availability. Operators may become unstable while handling equipment and cause foreign object debris (FOD) or damage to the hardware, structure, and personal injury during prelaunch task activities. The analysis described in this paper assesses motion during ground crew preflight tasks while the vehicle is oscillating and will be used for future vertically stacked vehicles.

\section{Research Focus}

Locations and configurations of the Ares I vehicle design were chosen for the analysis based on the most complete data available and information presented by the preliminary computerized Ares I Load Cell \#4 analysis, which provided motion data for a range of wind speed telemetries. ${ }^{1}$ According to the analysis, the vehicle and vehicle access arm (VAA) were both displaced; however, the VAA was stationary for this assessment. The assessment results are expected to be applicable to most future stacked launch vehicle designs.

The HF team focused the worksite analyses on the upper stage (US) maintenance access points consisting of the instrument unit (IU) and the interstage (IS) (Fig. 2). These analyses focused on the effects of sway on structural design, hardware safety, and operator efficiency. 


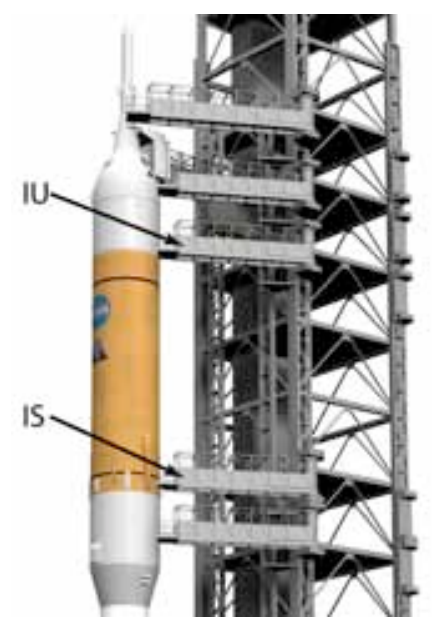

Figure 2. Ares I US IU and IS access points from the VAA.

This assessment focused on two prelaunch conditions: On-pad strong thunderstorms (180-day winds at $74 \mathrm{kn}$ ) and on-pad unfueled (1-day winds at $47 \mathrm{kn}$ ). Conditions were chosen based on the likelihood of ground crew operations taking place during the specified periods of time. It should be noted that this assessment was not intended to evaluate the effects of motion sickness on ground crew personnel nor on participant anthropometrics of range, force, or strength.

\section{A. Objective}

The primary objectives of this assessment consisted of physically simulating the oscillation envelopes initially identified and assessing the ability to carry out essential US operator tasks based on the predicted vehicle motion. ${ }^{1}$ Vehicle motion varied with peak wind speeds ranging from $34 \mathrm{kn}$ to $74 \mathrm{kn}$ in a thunderstorm. Alternating directional winds and wind gusts caused vortex shedding around the vehicle, creating the oscillating motion. ${ }^{1}$

\section{A. Methodology}

The HFE team led the part-task mockup configuration and integration and identified the operations tasks. MSFC's Flight Robotics Laboratory team managed the facility, tether system layout, and motion programming discussed in section III.

The Vehicle Displacement Analysis required reference materials compiled by using preliminary sources available at the time of the assessment. The Load Cell \# 4 analysis time history files recorded the motion and data taken from the Ares I IU and IS elevations depending on vehicle conditions, either with or without fuel, and various wind velocities. ${ }^{1} \mathrm{HF}$ analysis reports conducted at the Performance Analysis and Design Demonstrators (PADD) were used for guidance, procedures, requirements, and goals for each chosen task during the Vehicle Displacement Analysis.

Observations and comments recorded in the dynamic analysis were compared to observations and comments recorded of the same or similar tasks in a number of previously conducted static analyses. The areas of focus pertained to observations and human performance such as body posture, ability to maintain balance (standing still, moving, while holding/handling objects), number of times participant came in contact with access openings and surrounding hardware, ability to understand and carry out verbal instructions, ability to verbally communicate with each other and observers, and participant/observer perceived performance. Again, the assessment was not intended to evaluate anthropometric range, force, strength, or time to complete the task.

The following eight IU and IS maintenance and ground support operation tasks were chosen for analysis based on the fidelity of information/data available and comparison of prior assessments performed at the stationary/static PADD mockups.

1) Task 1: Simulate entry/exit of the IU.

2) Task 2: Simulate removal and replacement of a line replacement unit (LRU) on an IU avionics panel.

3) Task 3: Simulate removal and replacement of a battery LRU on an IU avionics panel by two participants.

4) Task 4: Simulate passing of internal access (IA) ground support equipment (GSE) through the IS access door. 
5) Task 5: Simulate loading of propellants into the roll control system (RoCS) panel, while an operator wore a self-contained atmospheric protection ensemble (SCAPE) suit.

6) Task 6: Simulate the installation of the IS flight door.

7) Task 7: Simulate arming the safe and arm (S\&A) device on an IU avionics panel.

8) Task 8: Simulate passing of IA GSE through the IU access door.

Participants were chosen based on past experience with flight hardware operations and technical knowledge of the vehicle design and maintenance tasks. They were classified according to stature per the requirement that the system shall provide worksites for launch site processing and maintenance tasks that are sized to be performed by ground crew with anthropometric dimensions for stature that are within the 5th to 95th percentiles of the worker population to stature, strength, and lifting. ${ }^{2}$ Although program requirements specified 5th percentile female up to 95th percentile male in stature, a 5th percentile female was not available for the assessment. Men were classified in the 95th stature percentile (referred to as the large participant) and women were classified in the 10th stature percentile (referred to as the small participant) or, in the case of Task 3, different combinations of both percentiles. ${ }^{3}$ This assessment did not include a person's strength or lifting ability.

Observers documented comments on each task concerning specific areas such as body clearance, tool clearance, stability, and posture. Remark sheets allowed areas of focus within each task and overall process to be rated on a five-point scale from excellent to poor. Different circumstances and task procedures required unique observation sheets for all eight tasks. Participants also recorded their opinions on similar observation sheets during an exit interview following each task. The full-scale, part-task mockup was designed to accommodate both the IU and IS task procedures.

\section{Configurations}

A description of the Flight Robotics Laboratory Facility, large mobility platform (LMP), part-task mockup layout and software programs follow. The mockup was constructed from the most recent drawings/information available for the access door(s), avionics panel, avionics boxes, and VAA. Flight-like hardware was used when available. The allowable tolerance was \pm 0.25 in for the US and the VAA mockups.

\section{A. Facility}

The MSFC Flight Robotics Laboratory Facility is the largest precision epoxy flat floor in the world. The floor lays within 0.001 in to the Earth's curvature allowing the 3,500-lb LMP to move in the desired oscillating motion. The reaction of the LMP to outside forces may differ from the flight vehicle's motion. ${ }^{4}$ This was not possible to confirm because human-induced vehicle motion data was not part of the preliminary analysis. The oscillating motion was slightly affected as a participant would push off of the LMP. It is not clear if this would occur with the launch vehicle.

The VAA mockup (represented by scaffolding) provided access through the vehicle's access door with a nominal distance of 6 in between the VAA and the LMP. A computer workstation was positioned near the edge of the flat floor to facilitate communication and maintain visual contact with the hardware.

\section{B. Large Mobility Platform Hardware}

The LMP floated 0.003 in above the flat floor on cushions of air supplied by three air-bearings (two on the front of the platform and one on the back as shown in Fig. 3). The design incorporated a simple single-pulley system (Fig. 4), a stability rope come-along, a manufactured fixture with four tether rings, guideline tubing, and a hard-line connection between the servo controller and the computer station. The pulley turned with the attached tether fixture to create a circular oscillation. Position holes across the pulley's diameter determined the total vehicle displacement, depending on the chosen radius. For example, when set in the 2 -in position, the vehicle's circular motion would produce a 4-in displacement. A jointed arm connected the vehicle and the flat floor's edge to provide additional stability in motion and prevent vehicle torque by reducing undue strain on the pulley tether system. Since the VAA was stationary, a 4-in displacement was used for this assessment. A displacement of 6 in or greater resulted in contact with the vehicle and VAA. 


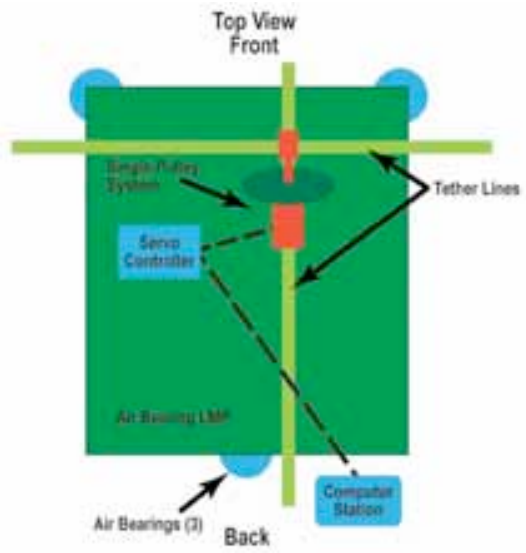

Figure 3. Final LMP configuration.

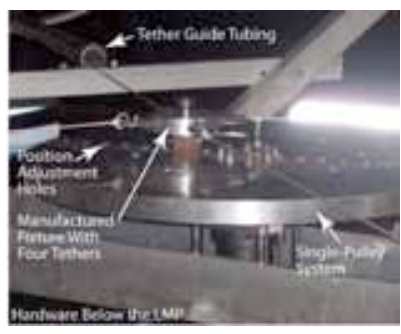

Figure 4. Single-pulley system.

\section{Mockups}

A wall segment (72 in high), representing US elevations at the IU and IS with appropriate hardware to simulate the chosen prelaunch task assessments, sat on top of the LMP. While facing the front of the LMP, a representative IU avionics panel was located just inside (inner mold line) on the left of the access door, and the IS RoCS service panel was located to the right of the door (outer mold line). A removable insert allowed the access door to function as both IU and IS access doors. The wall component simulated both the IU and IS hardware within the 9-ft $\times 9$-ft LMP. These features are shown in Figs. 5-7. A stationary mockup of the VAA was positioned 6 in from the moving platform and erected at the appropriate height for access. A 6-in grid was applied to the structure to assist in photographic and video documentation and to provide scale.

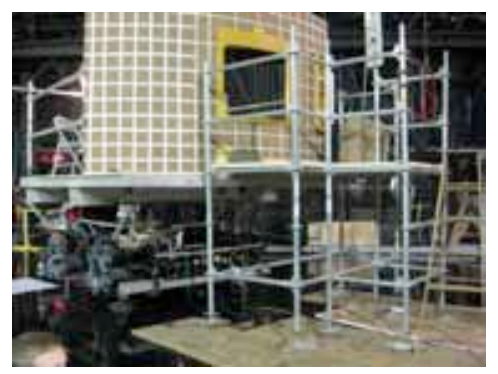

Figure 5. Front view of LMP and VAA. 


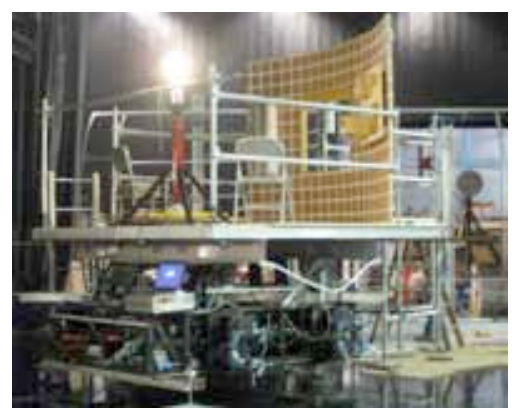

Figure 6. Back view of LMP and VAA.

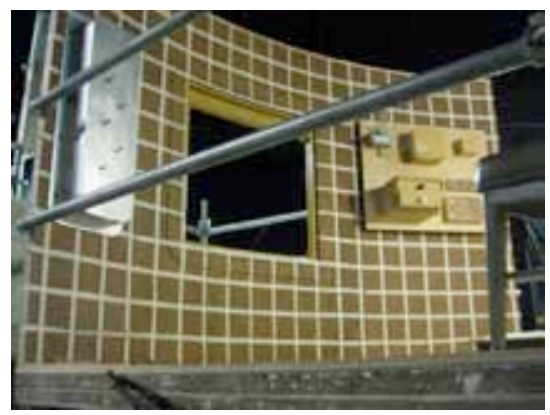

Figure 7. Side view of LMP with integrated hardware.

\section{Programming}

A laptop and computer workstation ran the Simulink programs that communicated through the controller to the two autonomous motors, producing the LMP movement. The two graphs in the controller display layout of Fig. 8 show the desired output motion of both motors. These graphs represent the approximate vehicle displacement through sinusoidal waves. A direct landline connection between the servo controller and the computers was used, but it did not impact platform movement.

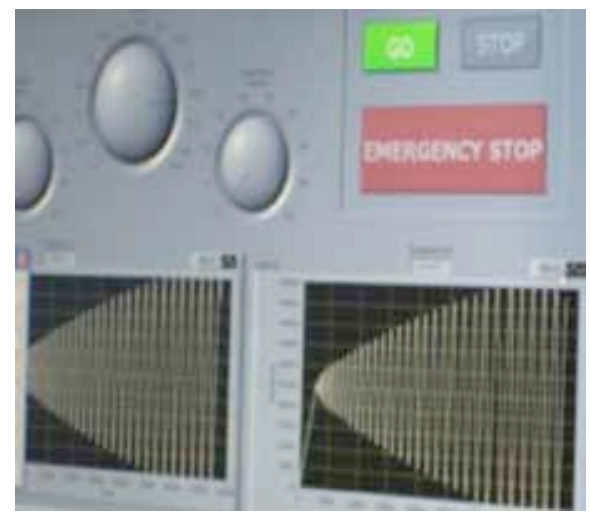

Figure 8. Program control panel. 


\section{Motion Test}

Eight tests were conducted to assess the capabilities of the vehicle's oscillation frequency and to demonstrate the vehicle's oscillating motion under each condition once the motor and controller system were tested with weights and integrated into the LMP. Tests were conducted at a 4-in radius (8-in diameter) displacement and at a 2-in radius (4in diameter) displacement. The 4-in displacement setting was used for the assessment.

\section{A. Vehicle Velocity Range}

The vehicle was unable to achieve the desired $0.5 \mathrm{~Hz}$ indicated in the computerized analysis. ${ }^{1}$ Motor and vehicle configurations were capable of reaching $0.4 \mathrm{~Hz}$; however, the desired platform motion was not achievable. As a result, the analysis was conducted at $0.15 \mathrm{~Hz}$ and $0.25 \mathrm{~Hz}$.

\section{B. Vehicle Oscillation}

Preliminary motion testing produced a crescent/triangular displacement. Further refinement of the platform pulley and tethering configuration resulted in an elliptical/circular motion. The progression of the vehicle's motion oscillations is illustrated in Fig. 9. The progression sequence was as follows: a) $0.15-\mathrm{Hz}, 8$-in displacement, b) $0.25-$ $\mathrm{Hz}$, 4-in displacement, c) $0.4-\mathrm{Hz}$, 4-in displacement, and d) $0.15-\mathrm{Hz}-0.25-\mathrm{Hz}$, 4-in displacement.
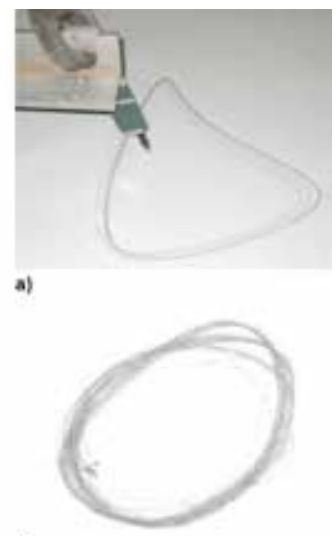

b)
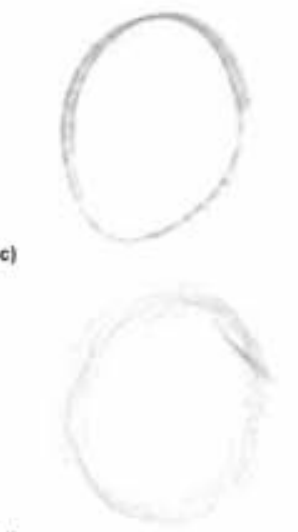

Figure 9. Test motion graph progression.

During a test demonstration at $0.15 \mathrm{~Hz}$ and $0.25 \mathrm{~Hz}$, a participant walked and stood on the moving platform for $\approx 10 \mathrm{~min}$. The circular motion was preserved; however, slight excursions or spikes in motion were observed while the participant walked across the platform. Following the simulation, the participant experienced residual sea-leg effects for $\approx 5$ min after exiting the vehicle. Residual sea-leg effects varied greatly with each individual. Some had 
no after effects after long exposure ( $>45 \mathrm{~min})$ to motion while others had symptoms after short periods $(<10 \mathrm{~min})$ of motion. The difference in frequency did not appear to be a factor in the after effects.

\section{Task Analysis}

Participants who performed each task were classified according to stature. Men were classified in the 95th percentile stature (referred to as the large participant) and women were classified in the 10th percntile stature (referred to as the small participant) or, in the case of Task 3, different combinations of two operators. ${ }^{2,3}$ This assessment was not inclusive of a participant's strength or lifting ability. Vehicle oscillations were alternated between $0.15 \mathrm{~Hz}$ and $0.25 \mathrm{~Hz}$ for each task and each participant for the eight tasks. The steps and procedures performed were recreated from previous analyses as closely as possible in order to compare the observations, participant comments, and participant performance, with motion being the only addition/variable to the tasks. In certain cases, it was not possible to recreate the physical environment completely, specifically within the IU, due to time constraints.

In certain cases, it was not possible to recreate the physical environment completely, specifically within the IU, due to time constraints.

\section{A. Task 1: Simulate Entry/Exit of the Instrument Unit}

Participants were observed as they entered/exited the IU volume through the access door (measuring 32 in high and 34 in wide) that was sized to accommodate the ground crew and various sizes, shapes, and weights of equipment, including LRU and IA GSE (which will be reviewed in tasks 2, 3, 4 and 8). ${ }^{5}$

Regardless of motion, contact with the doorframe was expected for stability. However, the motion and the door's height and distance above the VAA surface (19 in) contributed to the small participants contacting the vehicle doorframe with their back foot. A conscious effort was needed to maintain balance when the large participant passed through the door at $0.25 \mathrm{~Hz}$. Both large and small participants recommended the use of handholds/hand rails.

\section{B. Task 2: Simulate Removal and Replacement of the Line Replacement Unit on the Instrument Unit Avionics Panel}

Steps and procedures used in this task were provided from a previous IU analysis. ${ }^{6}$ The participants simulated the removal and replacement of a 10-1b, 5 -in $\times 7$-in $\times 4$-in avionics box on an avionics panel. Participants used a mirror and flashlight to verify that connector pins were visible through connector coupling ring inspection holes.

Participants suggested adding handles to LRUs to improve their ability to handle the hardware. In prior HF assessments, similar comments were made to include handholds on the LRUs. Both participant groups found that squatting required more concentration to maintain balance at the higher oscillation frequency. A smaller participant also used flight hardware for stabilization while standing and walking on the platform, which is typically discouraged due to potential hardware damage.

\section{Task 3: Simulate Removal and Replacement of the Avionics Battery Line Replacement Unit on the Instrument Unit Avionics Panel by Two Participants}

Steps and procedures used in this task were provided from a previous IU analysis. ${ }^{7}$ According to HF weight limitation requirements, the avionics battery (12 in $\times 8$ in $\times 7$ in and weighing $45 \mathrm{lb})$ must be handled by two operators or with assistive GSE. ${ }^{8}$ Participants located the battery on an IU avionics panel and used a socket wrench to manipulate the fasteners located on the battery to simulate battery removal and replacement.

While walking and working together, it was noted that both frequencies $(0.15 \mathrm{~Hz}$ and $0.25 \mathrm{~Hz})$ hindered the participants' ability to align the LRU with the mounting brackets. This task was repeated 10 times by each set of participants. A majority of time, the participants hit a side of the LRU mounting brackets before aligning and sliding the battery into place. Participants observed and reported that they would occasionally stumble as a result of the motion while walking backward.

\section{Task 4: Simulate Passing of Internal Access Ground Support Equipment Through the Interstage Access Door \\ Internal access GSE operation procedures were not clearly defined at the time of this assessment. For this task, the assumption was made that the IA GSE was passed between two operators into the vehicle and then out to the VAA (Fig. 10).}




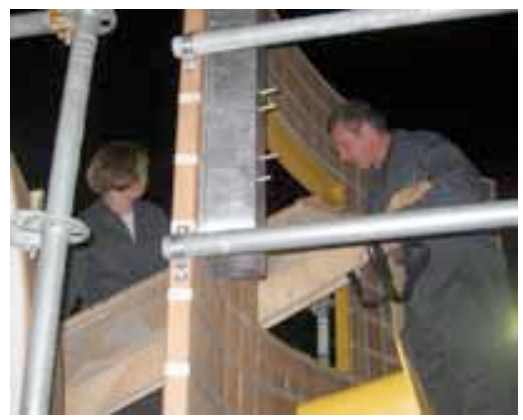

Figure 10. Small and large participants passing the IA GSE into the IS.

The GSE weight (35 lbs) and rectangular shape (60 in $\times 30$ in $\times 8$ in) was found to be cumbersome to handle, but the participants commented that the motion was only an issue when one person held the weight of the GSE. As a result, precautions (mainly slow methodical movement) were needed to prevent contact with the vehicle and surrounding hardware while handling the GSE.

\section{E. Task 5: Simulate Loading of Propellants Into the Roll Control System Panel While an Operator Wore a Self-Contained Atmosphere Protective Ensemble Suit}

The RoCS panel positioned outside the IS access door required the operator to perform the task from the VAA mockup. The participant wore a modified SCAPE suit without an active cryoair backpack, positive airflow, or faceplate. To recreate the task, procedures from a prior HF RoCS service panel analysis were used. ${ }^{9}$ The participant simulated fastening the flex hose to the flight service valve, actuating the service valve, and removal of the flex hose.

Only a large participant performed this task due to the available SCAPE suit size. The distance from the bottom of the RoCS service panel to the VAA platform surface was $14.3 \mathrm{in}$, requiring the participant to either kneel or squat to reach the lower connectors. Bending from the waste while in the SCAPE suit caused lower back strain due to the cryoair backpack weight. The confined space also prevented bending from the waist. The participant originally knelt on his knees, contacting the VAA surface, to mate the connecters. Although the participant stated that this provided the best sense of stability, the position was not acceptable for SCAPE operations due to the restricted air circulation within the suit and contact with potentially abrasive surfaces. The participant appeared unaffected by vehicle sway while performing the task while in a squatting position. The observers and participant noted that the space on the VAA structure was insufficient for SCAPE suit operations due to contact with the VAA railing to the right and behind the operator. In essence, the participant was able to stabilize himself against the structure while in the SCAPE, which is unacceptable. Insufficient VAA space had also been identified in the previous HF RoCS service panel assessment.

\section{F. Task 6: Simulate the Installation of the Interstage Flight Door}

Although flight door operation procedures were not clearly defined, the participants were instructed to use a speed wrench to remove the IS access door GSE while standing on the VAA and install the weighted (45 lbs) 36 -in $\times$ 36-in IS flight door.

It was observed that the space provided between the VAA scaffolding rail and the lower left hand side of the IS flight door was insufficient to properly use the speed wrench. The vehicle oscillation did not impact operator stability standing on the VAA but did amplify the potential contact of hardware or injuries due to the tight spaces (pinch points) of the VAA.

\section{G. Task 7: Simulate Arming the Safe and Arm Device on the Instrument Unit Avionics Panel.}

Participants followed procedures from a previous IU analysis to simulate IU S\&A device closeout. ${ }^{7}$ Vehicle sway appeared to have no affect on this task when compared to observations and participant comments from the previous analysis. Both participants stated that arming the S\&A device could be done at the higher frequency $(0.25$ $\mathrm{Hz}$ ). All participants carried out this task standing instead of the preferred seated position due to mockup limitations. 
It was observed that participants took a wider stance to compensate for motion and added stability when in the standing position. Although the mockup limitation changed the conditions of the task and performing the task while seating would greatly improve the work posture, there are other tasks that must be performed in the IU volume while standing. Due to motion, consideration for minimum IA GSE walkway widths should be addressed if the operators are required to widen their stance for stability.

\section{H. Task 8: Simulate Passing of Internal Access Ground Support Equipment Through the Instrument Unit Access Door.}

Although IA GSE operation procedures were not clearly defined, the participants (standing on either side of the door) passed the IA GSE wedges to each other into the moving vehicle and then out to the stationary VAA.

The wedge shape and overall size of the GSE (27 in $\times 25$ in $\times 20$ in) was found to be awkward to handle; however, participants noted that the biggest concern was holding all of the weight (35 lbs) inside the moving vehicle. This task was found to be more feasible for the larger participant. The smaller participant stated that they felt unstable at $0.25 \mathrm{~Hz}$.

\section{Analysis Conclusions}

Information gathered during the assessment indicated that motions as low as $0.25 \mathrm{~Hz}$ hampered the balance and performance of the ground crew personnel. Although the tasks could be performed at this frequency, the operators' ability to focus completely on the task was compromised. Observed changes in a participant's body position and stance revealed a need to accommodate for motion to maintain balance. Although slight stumbles or contact with flight hardware were noted in the static assessments, they were more prevalent in the dynamic assessments. To carryout tasks at the $0.5-\mathrm{Hz}$ threshold will provide significant challenges. If operators will be expected to perform tasks at these higher frequencies, handholds, tethers, tools, spacing (vehicle to service structure), IA GSE size, shape, and weight are potential design issues that will need to be considered with vehicle motion in mind.

Tasks performed from the service structure at the vehicle outer mold line should accommodate for dynamic work envelopes and work sites. What appeared to be an adequate work envelope for installation of the vehicle flight door on paper was found to be marginal in certain locations due, in part, to the dynamic envelope.

Many of the participant comments and observations recorded for this assessment were documented in previous assessments, which indicates several of the operational issues associated with the selected tasks were not due to vehicle motion but with vehicle and service structure configuration. These work volumes are not designed for human presence. They are built for the purpose of propulsion, although human presence is required to complete critical vehicle preparation and closeout tasks or to perform maintenance activities. Unlike crew capsules that provide seating, controls, displays, lighting, etc., these volumes do not provide a human friendly environment and must be designed to provide adequate protection of extremely sensitive flight critical hardware.

The ground crew is required to install removable access platforms and handholds, carry portable test equipment, provide their own lighting, and possess a host of various sized tools (all considered FOD) that must be designed appropriately to perform tasks in these confined areas in a manner that protects the ground crew and critical flight hardware. This is a demanding time-consuming challenge while the vehicle is motionless, and added vehicle motion only exacerbates the already challenging circumstances.

The participants for this sway assessment, who were involved with previous static assessments, noted that their overall performance was perceived to be diminished due to the effects of working on a moving vehicle and that they were unable to focus on the task at the same level as performed on the static mockup. Although there was no time duration recorded for the tasks in either the static or sway assessment, participant perceptions of slowed performance should be considered as a negative impact to ground crew performance.

As mentioned in section $\mathrm{V}$, it was not possible to recreate the physical environment due to time constraints. It should also be noted that the platform (a single-level flat surface) on which the participants stood while inside the mockup provided far more flat surface area $(8 \mathrm{ft} \times 8 \mathrm{ft})$ than the minimum requirement of 27 in $\times 25$ in as provided by the (multilevel/stepped) IA GSE concept. This may have played a far more critical roll in the assessment due to the observed change in widened stance, prohibiting the participant from widening their stance while perpendicular to the inner mold line.

These observations are just some of the challenges that will need to be addressed in order to improve the processes for ground operations required to maintain the unique vehicles and associated infrastructures currently under consideration. In order to streamline the operational life cycle of new launch vehicles, it is important to consider design nuances associated with vehicles other than what has been experienced over the past 30 years with the shuttle program. Lessons learned from past vehicles are important and must be addressed but, in some cases, 
lessons learned may not be applicable to new vehicle configurations. New technologies needed to increase reliability and mission availability for launch vehicles should also apply to ground infrastructure and ground crew performance. This may require additional mass allocation to the vehicle for internal access interfaces such as handholds and tethering points and evaluation of the ground support structures used to service the vehicle to reduce or eliminate vortex shedding around the vehicle creating the oscillating motion.

\section{Future Analysis}

In order to perform a more thorough assessment in the future, the mockup should include an independently moving VAA platform, and be capable of achieving the $0.5 \mathrm{~Hz}$ and maximum displacement to replicate the data more accurately. Since most of the tasks performed took place in under $30 \mathrm{~min}$ with several breaks, future assessments should include the effects of sway on the operator's physical response and ability to perform prelaunch tasks for periods of time lasting $1 \mathrm{hr}-3 \mathrm{hr}$ and full 8 -hr days with typical breaks. This would provide data needed to baseline the velocity and range of motion an individual can efficiently work for a known duration of time. Participants would begin performing tasks at $0.5 \mathrm{~Hz}$ or higher working back to an appropriate frequency for the ground crew operator to tolerate. Once a safety margin is factored into this baseline frequency, time duration studies would allow future guidelines for safe ground crew work areas and optimum shift times.

\section{Acknowledgments}

A special thanks is given to the following team participants and those who provided resources.

\section{MSFC EV82 Team}

Don Krupp

Charlie Dischinger

John Smith

David Reynolds

Clay Robertson

Chris Daniels

Richard Howard

Fred Flack

\section{MSFC ES32 Team}

Tom Bryan

Ricky Howard

Thomas Perrin

Charles Cowen

Preliminary Report

Dr. Steve Hahn

Thomas Perrin

\section{Other Resources}

Allen Taylor

Charlie Cox

Stephen V. Fotovich, Jr

\author{
Branch Chief \\ Human Factors Team Lead \\ Shop Manager, Mockup Lead \\ Mentor \\ Mentor \\ CADD Models \\ IU GSE CADD Models \\ SBU review
}

\author{
Team Co-Lead \\ Team Co-Lead \\ Programming Lead \\ Manufacturing Lead
}

Dynamic Concepts

MSFC EV91

Functional Task Objective Sheets

MSFC Repository

Technical Writer/Editor

\section{References}

\footnotetext{
${ }^{1}$ Hahn, S., "LC4 Prelaunch Loads Delivery.” DCI-Tm-012309-1047-02, Dynamic Concepts, Inc, Huntsville, AL, January 2009.

2“Constellation Program Human-Systems Integration Requirements,” CxP 70024, Marshall Space Flight Center, AL, March 2008.
} 
${ }^{3}$ Gordon, C.C., Chruchill, T., Clauser, C.E., et al.,"1988 Anthropometric Survey Of U.S. Army Personnel: Summary Statistics (ANSUR)," Natick/TR-89/027, United States Army Natick Research, Development and Engineering Center, Natick, MA, March 1989

${ }^{4}$ Roe, F. D., Gray, S. L., and Randall, J.L., "MSFC Flight Robotics Laboratory Description,” Marshall Space Flight Center, AL, 1996.

${ }^{5}$ Blume, J., "Minimum Hatch Clearance Requirement," ESTSG-FY10-00036, Jacobs ESTS Group, Huntsville, AL, March 2010.

${ }^{6}$ Blume, J., "Instrument Unit Avionics Logistics Candidate Item Access," HFSE-US-REP-016, Jacobs ESTS Group, Huntsville, AL, May 2008

${ }^{7}$ Krupp, D., "IU FTS VAB Battery Installation and Pad Operations," ESTSG-FY08-00807, Jacobs ESTS Group, Huntsville, AL, April 2008

${ }^{8}$ Blume, J., “Ares I Upper Stage Element Human Factors Engineering Requirements," Ares-USO-LS-25404, Marshall Space Flight Center, AL, March 2010

${ }^{9}$ Bolton, K., "RoCS Analysis," HFE-US-REP-027, Stage Analysis Branch, Marshall Space Flight Center, AL, May 2010 (unpublished) 